\title{
Sound-Evoked Olivocochlear Activation in Unanesthetized Mice
}

\author{
Anna R. Chambers ${ }^{1,2}$, Kenneth E. Hancock ${ }^{1,3}$, Stéphane F. Maison ${ }^{1,3}$, M. Charles Liberman ${ }^{1,3}$, \\ and Daniel B. Polley ${ }^{1,2,3}$ \\ ${ }^{1}$ Eaton-Peabody Laboratory, Massachusetts Eye E Ear Infirmary, 243 Charles St., Boston, MA 02114, USA \\ ${ }^{2}$ Program in Neuroscience, Harvard Medical School, Boston, MA 02115, USA \\ ${ }^{3}$ Department of Otology and Laryngology, Harvard Medical School, Boston, MA 02114, USA
}

Received: 5 October 2011; Accepted: 8 November 2011; Online publication: 13 December 2011

\begin{abstract}
Genetic tools available for the mouse make it a powerful model to study the modulation of cochlear function by descending control systems. Suppression of distortion product otoacoustic emission (DPOAE) amplitude by contralateral acoustic stimulation (CAS) provides a robust tool for noninvasively monitoring the strength of descending modulation, yet investigations in mice have been performed infrequently and only under anesthesia, a condition likely to reduce olivocochlear activation. Here, we characterize the contralateral olivocochlear reflex in the alert, unanesthetized mouse. Head-fixed mice were restrained between two closed acoustic systems, while an artifact rejection protocol minimized contamination from self-generated sounds and movements. In mice anesthetized with pentobarbital, ketamine or urethane, CAS at $80 \mathrm{~dB}$ SPL evoked, on average, a $<1$-dB change in DPOAE amplitude. In contrast, the mean CASinduced DPOAE suppression in unanesthetized mice was nearly $8 \mathrm{~dB}$. Experiments in mice with targeted deletion of the $\alpha 9$ subunit of the nicotinic acetylcholine receptor confirmed the contribution of the medial olivocochlear efferents to this phenomenon. These findings demonstrate the utility of the CAS assay in the unanesthetized mouse and highlight the adverse effects of anesthesia when probing the functional status of descending control pathways within the auditory system.
\end{abstract}

Correspondence to: Anna R. Chambers · Eaton-Peabody Laboratory, Massachusetts Eye \& Ear Infirmary · 243 Charles St., Boston, MA 02114, USA. Telephone: +1-617-3915953; fax: +1-617-7204408; email: Anna_Chambers@meei.harvard.edu
Keywords: olivocochlear, corticofugal, arousal, attention, anesthesia, contralateral reflex, outer hair cell

\section{INTRODUCTION}

The mammalian auditory system features three major networks of descending projections: (1) corticofugal projections from the auditory cortex to thalamic, midbrain, and brainstem nuclei (Suga et al. 2002; Winer 2006); (2) olivocochlear projections from neurons in the superior olivary complex to the cochlea (Guinan 2006); and (3) reflex pathways mediating sound-evoked contractions of the middle ear muscles (Mukerji et al. 2010). Collectively, these descending pathways are believed to mediate a number of adaptive phenomena such as signal-innoise detection (de Boer and Thornton 2008), tuning specificity (Bauerle et al. 2011; Yan et al. 2005), experience-dependent perceptual reweighting (Bajo et al. 2010), and protection of the inner ear from acoustic trauma (Lauer and May 2011; Maison and Liberman 2000). Of the three systems, the olivocochlear projections are the best understood, thanks in part to recent studies that have taken advantage of the genetic tools uniquely available in the mouse. Studies of mutant mice have clarified the molecular mechanisms underlying synaptic transmission between the medial olivocochlear (MOC) terminals and outer hair cells (OHCs; Maison et al. 2007; Taranda et al. 2009; Vetter et al. 1999, 2007), the role of MOC feedback in reducing acoustic injury (Lauer and May 2011; 
Maison and Liberman 2000; Maison et al. 2002), and the embryonic development of olivocochlear projections (Rossel et al. 2005).

While the genetic tractability of the mouse has aided the study of molecular mechanisms underlying olivocochlear function, integrative studies of the olivocochlear system in mice are uncommon. The functional status of MOC efferents has been noninvasively assayed in many other mammals through measurement of the MOC reflexes in which the amplitude of otoacoustic emissions are modulated upon sound presentation to the contralateral or ipsilateral ear (Boyev et al. 2002; Harrison et al. 2008; Henin et al. 2011; Moulin et al. 1993). However, reduced otoacoustic emission amplitudes following contralateral acoustic stimulation (CAS) in mice have only been reported by one group (Frisina et al. 2007; Zhu et al. 2007), and the role of the MOC system rather than other feedback systems such as the middle ear muscle reflex has not been directly demonstrated. In addition, olivocochlear reflex strength in mice has only been studied under anesthesia, a condition likely to reduce the excitability of MOC neurons, thereby interfering with the MOC-mediated inhibition of OHCs (Avan et al. 1996; Boyev et al. 2002; Guitton et al. 2004; Kujawa and Liberman 2001; Liberman and Brown 1986). Therefore, an effective method for reliably and noninvasively measuring MOC efferent reflexes in unanesthetized mice is of interest.

Here, we describe a technique for measuring the contralateral MOC reflex in unanesthetized, headrestrained mice. We directly test the hypothesis that anesthesia greatly attenuates the MOC reflex by measuring modulation of ipsilateral DPOAE amplitude by CAS in unanesthetized mice compared with that observed with common veterinary anesthetics: pentobarbital sodium, ketamine, and urethane. To test for the direct involvement of MOC efferents, CASinduced DPOAE suppression was measured in mice expressing a null mutation of the $\alpha 9$ nicotinic acetylcholine receptor in which OHCs are rendered insensitive to MOC input (Vetter et al. 1999). Our findings demonstrate that the unanesthetized mouse can be effectively used for MOC reflex measurements and further suggest that the origin of DPOAE suppression previously reported in the anesthetized mouse requires reconsideration.

\section{METHODS}

\section{Animal preparation}

All procedures were approved by the Massachusetts Eye and Ear Infirmary Animal Care and Use Committee and followed the guidelines established by the National Institutes of Health for the care and use of laboratory animals. Experiments were performed on adult (8-10 weeks old) female $\mathrm{CBA} / \mathrm{CaJ}(N=24)$ and $\alpha 9^{-/-}$and $\alpha 9^{+/+}$mice ( $N=3$ and 4, respectively), which were a 129-CBA/CaJ hybrid (Vetter et al. 1999). All experiments were conducted in a doublewalled sound-attenuating chamber, with core body temperature maintained at $37.5^{\circ} \mathrm{C}$ in both anesthetized and unanesthetized mice via a homeothermic blanket system (FST). Anesthesia was induced using one of the following protocols: (1) ketamine (100 mg/kg, i.p.) and xylazine (10 mg/kg, i.p.); (2) pentobarbital $(50 \mathrm{mg} / \mathrm{kg}$, i.p.) with chlorprothixene $(30 \mathrm{mg} / \mathrm{kg}$, i.m.); or (3) urethane $(1.2 \mathrm{~g} / \mathrm{kg}, \mathrm{i} . \mathrm{m}$.) and xylazine $(20 \mathrm{mg} / \mathrm{kg}$, i.p. $)$.

For unanesthetized experiments, a titanium headplate, $37.3 \times 5.2 \times 0.9 \mathrm{~mm}(L \times W \times H)$ weighing $0.5 \mathrm{~g}$, was first affixed to the skull under anesthesia (see Wienisch et al. 2011). The dorsal surface of the skull was exposed, a thin layer of cyanoacrylate (VetBond, WPI, Inc.) applied, and the headplate affixed to the dorsal aspect of the skull near bregma with acrylic bonding material (C\&B Metabond). Tissue adhesive (VetBond, $3 \mathrm{M}$ ) was applied to the wound margin. Postoperative subcutaneous injections of buprenorphine $(0.05 \mathrm{mg} / \mathrm{kg})$ and saline $(0.5 \mathrm{ml})$ were given to reduce pain and dehydration, respectively. Data for the ketamine/xylazine condition were collected at the conclusion of the headplate fixation procedure. These same mice were subsequently tested in the unanesthetized condition following at least $48 \mathrm{~h}$ of recovery. Measurements made under urethane/xylazine and pentobarbital/chlorprothixine anesthesia were terminal procedures.

Mice were briefly sedated with $2-5 \%$ isoflurane $(<5 \mathrm{~min})$ shortly before unanesthetized recordings to allow inspection of the ear canal and attachment of pinna inserts for sound delivery. During ear canal inspection for all animals, a small slit was made at the base of the pinna to better visualize the tympanic membrane. Funnel-shaped plastic inserts $(1.2 \mathrm{~cm}$ long, 4.3- and 1.4-mm diameter for the ends facing the speaker assembly and the tympanic membrane, respectively) were affixed to the external auditory meatus with a cyanoacrylate gel. Mice were blinking, whisking, and grooming (i.e., awake) within $30 \mathrm{~s}$ following cessation of isoflurane. They were allowed to recover for an additional $10-20 \mathrm{~min}$ as the equipment was set up and calibrated. Experiments were not initiated until mice appeared fully alert, as confirmed through visual monitoring. Subsequent visual monitoring during the experiment via an infrared camera confirmed that mice remained awake during all unanesthetized experiments. All experiments were conducted in the dark. With head fixation, light tail restraint on the heating pad, and bilateral acoustic assemblies positioned in the ears, 
most mice remained still, with only brief periods of movement for 30-40 $\mathrm{min}$.

\section{DPOAE suppression assay}

Acoustic assemblies consisted of two miniature dynamic earphones used as sound sources (CUI CDMG15008-03A) and an electret condenser microphone (Knowles FG-23329-PO7) coupled to a probe tube to measure sound pressure near the eardrum. Stimuli were generated digitally, and sound pressure was amplified and digitally sampled at $5 \mu$ s. The probe tube microphone was calibrated in a small coupler with a 0.25-in. condenser microphone positioned where the eardrum would be during physiological testing. Primary tones were kept at a constant ratio $\left(f_{2} / f_{1}=1.2\right) ; f_{2}$ was presented at $10 \mathrm{~dB}$ below the $f_{1}$ level and the resultant DPOAE measured at $2 f_{1}-f_{2}$. Each ear canal SPL measurement was obtained from $1.6 \mathrm{~s}$ of spectral and waveform averaging.

To measure the modulation of DPOAEs by contralateral noise, DPOAEs with $f_{2}$ at $11.3,16$, or $22.6 \mathrm{kHz}$ were continuously measured before, during, and after continuous CAS. The primary tone level was set to elicit a $2 f_{1}-f_{2}$ distortion product amplitude approximately $10 \mathrm{~dB}$ greater than the noise floor, which was achieved by presenting the $f_{2}$ levels at $35.7 \pm 5.8 \mathrm{~dB}$ SPL $(11.3 \mathrm{kHz}), 19.1 \pm 8.2 \mathrm{~dB}(16 \mathrm{kHz})$, and $39.7 \pm$ $9.6 \mathrm{~dB}(22.6 \mathrm{kHz})$. The primary levels used to elicit the DPOAE did not vary between anesthetic conditions (Mann-Whitney $U$ tests, $p>0.05$ ). CAS consisted of continuous white noise delivered through an identical acoustic system at $80 \mathrm{~dB}$ SPL for up to $480 \mathrm{~s}$ (300 DPOAE measurements). In the study of $\alpha 9-$ null and wild-type littermates, where the time course of the suppression over hundreds of trials was not the focus of investigation, CAS was shortened to 24-32 s. The absence of acoustic cross talk was confirmed by monitoring changes in the acoustic noise floor in both ear canals and lowering the CAS by up to $10 \mathrm{~dB}$, as necessary.

\section{Artifact rejection in DPOAE testing}

Movement-generated artifacts are a challenge when recording low-level acoustic signals from awake animals. Visual monitoring confirmed that sudden changes in the level of the primaries or the noise floor (Fig. 1A) usually corresponded to movementinduced misalignment of the acoustic assembly and/ or movement-induced acoustic contamination. In the present experiments, $<30 \%$ of all trials were judged to contain movement artifacts (Fig. 1B), and $>50 \%$ of contaminated trials were from one animal, subject no. 5 . To minimize movement-related changes in stimulus amplitude, we implemented a continuous recalibration

algorithm: during each DPOAE measurement, the $f_{1}$ and $f_{2}$ levels were measured and then corresponding earphone voltages adjusted on the subsequent trial to produce the desired SPLs. We interleaved measurements with and without recalibration in subject no. 5 and confirmed that recalibration could increase the percentage of trials retained (Fig. 1C, dashed line) without affecting the level of maximum DPOAE suppression (Fig. 1C, solid line).

Elimination of movement artifacts from the DPOAE data was accomplished by dropping individual measurements in which either (1) $f_{1}$ and $f_{2}$ levels deviated by more than $\pm 1 \mathrm{~dB}$ from their specified amplitudes or (2) noise floor values deviated significantly from the mean. All remaining measurements were then normalized to the mean DPOAE amplitude recorded during the preCAS period. DPOAE amplitudes were calculated by grouping DPOAE measurements into 8-s bins (five measurements per bin) relative to CAS onset. The magnitude of DPOAE suppression was defined either as the maximum change in DPOAE amplitude, which differentiates facilitative and suppressive effects with positive and negative signs, respectively (Figs. 1 and 2A), or the absolute value of maximum DPOAE amplitude change, which describes the magnitude of amplitude

A
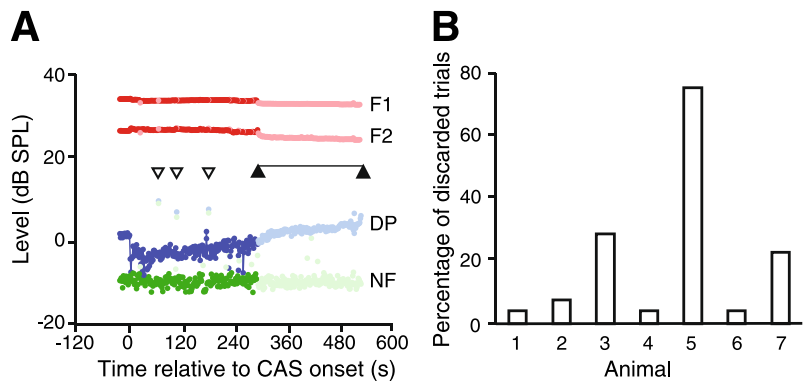

C

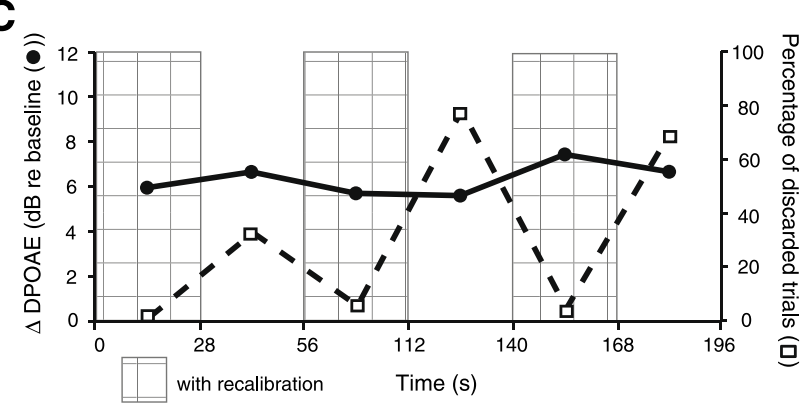

FIG. 1. Movement artifacts during DPOAE measurements in unanesthetized mice. A Animal movement introduced artifacts (lighter points) in the primary (filled triangles) or noise floor (open triangles) levels. B Percentage of discarded trials for each animal tested in the unanesthetized case, before dynamic recalibration. Values represent the mean of three runs, once at each tested frequency. C Example mouse in which runs with (hatching) and without dynamic recalibration were interleaved. Dynamic recalibration decreased the percentage of discarded trials (dotted line) without affecting the maximum CAS-induced DPOAE suppression (solid line). 

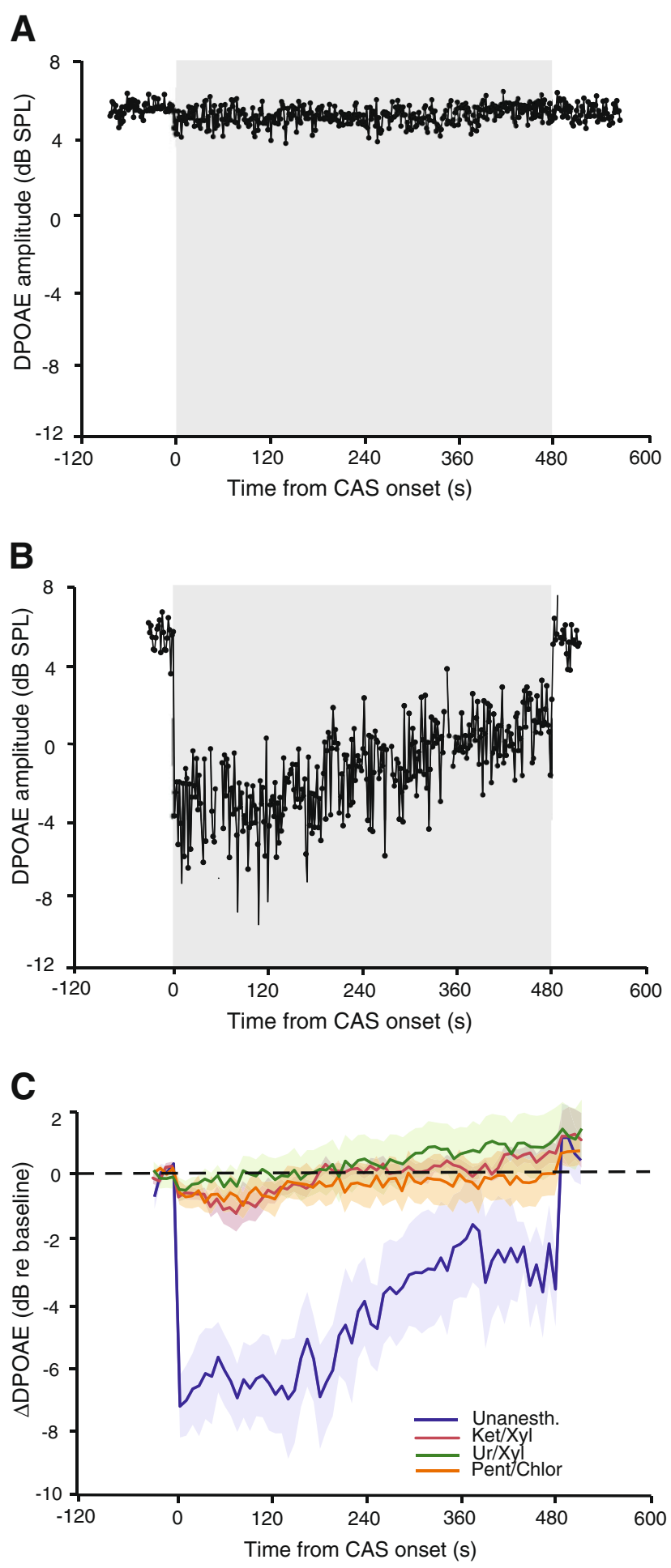

FIG. 2. Amount of CAS-evoked DPOAE suppression depends on state of arousal. A Contra-noise suppression of the DPOAE $\left(f_{2}=16 \mathrm{kHz}\right.$, $L_{2}=25 \mathrm{~dB} \mathrm{SPL}$ ) under ketamine/xylazine anesthesia. Gray box indicates timing of contralateral broadband noise presentation (80 dB SPL). B Contra-noise suppression of DPOAE from the same mouse before anesthetization. The same stimulus conditions are used for (A) and (B). C Mean change in DPOAE amplitude relative to the pre-CAS baseline ( $n=$ 8 for unanesthetized and ketamine/xylazine groups, $n=4$ for urethanexylazine and pentobarbital groups). Unanesthetized and ketamine/ xylazine groups were the same eight animals. In (C) only, each point is an average of five consecutive time points; shaded areas indicate SEM. change regardless of sign (Fig. 2B, C). Data are described as the means \pm SEM and analyzed with an ANOVA when data samples met the assumptions for parametric statistics. In cases where they did not (e.g., by failing to conform to a normal distribution), central tendencies of each sample were described according to the median value and inferential statistics were carried out with nonparametric tests.

\section{RESULTS}

To assess the effect of anesthesia on the strength of the MOC sound-evoked reflex, ipsilateral DPOAEs were elicited with low-level probe tones and the amplitude of the $2 f_{1}-f_{2}$ distortion product measured before, during, and after CAS. The intensity and spectra of the CAS was selected to effectively drive MOC efferents without introducing acoustic cross talk between the sound delivery systems. The duration of the CAS ( $8 \mathrm{~min})$ was set to allow us to study both the fast suppressive MOC effects, which occur within hundreds of milliseconds and are mediated by wellstudied cholinergic effects on OHCs (Fuchs and Murrow 1992), as well as slower effects, both suppressive (Sridhar et al. 1995) and facilitative (Maison et al. 2007), that occur over tens of seconds (when the MOC activity is shock-evoked) and arise from mechanisms that are poorly understood.

As reported by others (Zhu et al. 2007), when mice were anesthetized, CAS elicited only a small change in the ipsilateral DPOAEs. In the example from a ketamine/xylazine-anesthetized mouse shown in Figure 2A, the effect of CAS onset is barely detectable in the ipsilateral DPOAE elicited with $f_{2}$ at $16 \mathrm{kHz}$, and no further change is apparent at CAS offset. In contrast, in the same mouse when alert and unanesthetized (Fig. 2B), CAS elicited a large $(\sim 10 \mathrm{~dB})$ reduction in the ipsilateral DPOAE amplitude. Although the amount of suppression decreased by $\sim 5 \mathrm{~dB}$ over the 8-min CAS period, there was an abrupt recovery to pre-noise baseline immediately upon termination of the CAS.

The mean data (Fig 2C) illustrate the dramatic difference in CAS-evoked suppression for the anesthetized vs. unanesthetized conditions. In the unanesthetized mice, the CAS produced an immediate DPOAE suppression of almost $8 \mathrm{~dB}$, on average. As the CAS continued, the suppression decreased ( $F=$ 5.813, $p<0.001$, within-subject ANOVA). At CAS offset, the mean DPOAE immediately returned to a level close to the pre-CAS baseline. As shown in Figure 2C, three different anesthetic regimens were compared: ketamine/xylazine, urethane/xylazine, and pentobarbital/chlorprothixene. The effect of CAS on DPOAE amplitude under anesthesia was small $(<1 \mathrm{~dB})$, but 
changed significantly across the CAS period for ketamine/xylazine $(F=4.42, p<0.001)$ and urethane/ xylazine $(F=2.73, p<0.001)$, although not for pentobarbital/chlorprothixene $(F=1.2, p>0.05)$. Although the differences between the unanesthetized and combined anesthetized conditions were highly significant $(F=8.35, p<0.001$, between-subject ANOVA), the overall differences were not observed between the three anesthetic conditions (ANOVA, $F=0.6, p>0.05$ ).

In experiments where MOC efferents are directly activated through the electrical stimulation of the olivocochlear bundle, suppressive effects on DPOAEs vary according to the primary tone frequency (Maison et al. 2007). As shown in Figure 3A, in awake animals, the magnitude of CAS-evoked suppression was similar for DPOAEs elicited with $f_{2}$ at 11.3 and $16 \mathrm{kHz}$, but slightly, though not significantly, smaller for $f_{2}$ at $22.6 \mathrm{kHz}(p>0.05$, Wilcoxon signed-ranks test). Although CAS onset in awake mice always elicited DPOAE suppression, CAS effects in anesthetized mice could be either the suppression or facilitation of ipsilateral DPOAE amplitudes (Fig. 3A). The changes, although small, could be greater than the baseline fluctuations seen prior to CAS onset and, therefore, appears related to the CAS. A tendency for facilitation was also seen following CAS offset; however, the offset effect did not reach significance (Wilcoxon signedranks tests for pre-CAS vs. post-CAS, $p>0.05$ for each $f_{2}$ frequency and anesthetic condition; Fig. 3A). Given that CAS induced either facilitation or suppression under anesthesia, we further analyzed the data using the absolute value of the change in DPOAE amplitude (Fig. 3B). The absolute values of maximum of the DPOAE amplitude changes differed significantly from the pre-CAS period across all $f_{2}$ frequencies and anesthetic conditions (Wilcoxon signed-ranks test, $p<0.05$ for each $f_{2}$ frequency; Fig. 3B). However, the absolute values of DPOAE amplitude changes were significantly greater in the unanesthetized condition than in each of the anesthetized conditions (MannWhitney $U$ tests for each frequency between unanesthetized vs. ketamine/xylazine $(p<0.001)$, urethane/ xylazine $(p<0.01)$, and pentobarbital/chlorprothixene $(p<0.01)$ are all significant after correcting for multiple comparisons; Fig. 3B). Absolute DPOAE amplitude changes between anesthetic conditions did not significantly differ from each other (Mann-Whitney $U$ tests, $p>0.05$ for all comparisons).

To assess the adaptation of CAS-induced effects, we compared the maximum DPOAE changes in the first and the second halves of the 8-min CAS period (Fig. 3C). In anesthetized mice, the time course patterns averaged across all $f_{2}$ frequencies were heterogeneous: DPOAE amplitudes sometimes increased, sometimes decreased, and sometimes stayed constant during the noise (Wilcoxon signed-ranks test, $p>0.05$ for
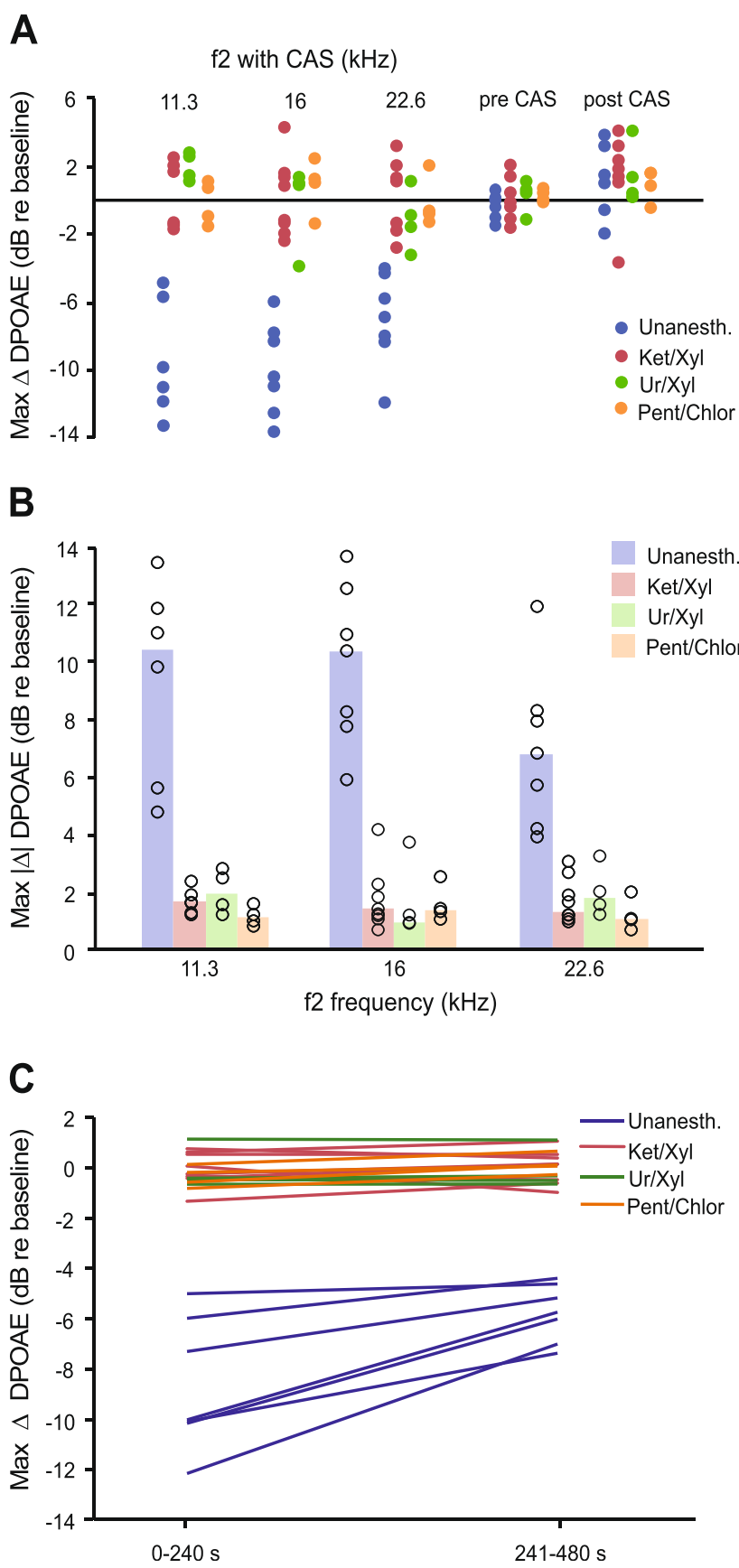

FIG. 3. CAS-evoked DPOAE suppression is stronger and more stereotyped in unanesthetized mice. A Dot density graph of the maximum CAS-induced DPOAE change in individual mice at three ipsilateral $f_{2}$ frequencies. Pre- and post-CAS refer to measurements made at $f_{2}=16 \mathrm{kHz}$ before and after CAS, respectively, to indicate fluctuations in baseline DPOAE amplitude. B Absolute values of CAS-induced maximum DPOAE change in individual mice shown as open circles; bars represent the median. C Absolute value of the maximum CAS-induced DPOAE suppression during the first and second halves of noise presentation for each mouse.

all groups). However, unanesthetized animals displayed a stereotyped sign (suppression) and time course: effect size always decreased from the first to second half of the CAS (Fig. 2C; Wilcoxon signed-ranks test, $p<0.001$ ). 
To determine the contribution of MOC efferents to the large CAS effect observed in the unanesthetized state, we studied mice with targeted deletion of the $\alpha 9$ nicotinic acetylcholine receptor. Such mice are functionally de-efferented (Vetter et al. 1999) because all classic MOC effects on OHCs require receptors including the $\alpha 9$ subunit. As shown in Figure 4, unanesthetized $\alpha 9^{-/-}$mice exhibited a substantially reduced DPOAE suppression when compared with wildtype littermates or wild-type CBA/CaJ mice (MannWhitney $U, p<0.01$; Fig. 4). Significant differences in DPOAE suppression between the wild-type mice of the two strains were not observed (Mann-Whitney $U$, $p=0.3$ ). These experiments confirmed that most of the CAS-induced DPOAE suppression observed in unanesthetized mice arose from synaptic coupling between MOC fibers and $\alpha 9 / \alpha 10$ AChRs in OHCs. The residual DPOAE suppression observed in $\alpha 9^{-/-}$mice is roughly equivalent to levels of DPOAE suppression observed in anesthetized wild-type mice. The results are consistent with the idea that much of the CAS effect seen in anesthetized mice may arise from sources other than the MOC system. These issues have been addressed in a recent abstract (Maison et al. 2011).

\section{DISCUSSION}

Sound-evoked vs. shock-evoked olivocochlear activity in mice

In anesthetized animals, the OC system can be activated by shocks delivered to the OC bundle, at the floor of the fourth ventricle (Wiederhold and Kiang 1970). In CBA/CaJ mice, the strain used in the present study, the presentation of long shock trains $(70$ s) to the OC bundle (Maison et al. 2007) suppresses ipsilateral DPOAEs in a pattern that is qualitatively similar to that seen in the present study, in which OC activity is elicited by CAS; that is, (1) significant suppression is seen in the first measurement point after onset of the OC-elicitor, (2) maximum suppression is seen within the first few seconds, (3) the amount of suppression decreases as the elicitor stimulus (shocks or CAS) continues, and (4) an enhancement effect is seen whereby, after offset of the elicitor stimulus, the DPOAE amplitude is larger than that seen before the elicitor onset. Prior work

FIG. 4. CAS-induced DPOAE suppression in the unanesthetized mouse is largely, though not completely, mediated by the MOC system. A Example trace from an unanesthetized $\alpha 9^{-/-}$mouse $\left(f_{2}=\right.$ $16 \mathrm{kHz}, L_{2}=35 \mathrm{~dB} \mathrm{SPL}, \mathrm{CAS}$ level $\left.=80 \mathrm{~dB}\right)$. B Example trace from an unanesthetized $\alpha 9^{+/+}$mouse $\left(f_{2}=16 \mathrm{kHz}, \mathrm{L}_{2}=33 \mathrm{~dB}\right.$ SPL, CAS level $=$ $80 \mathrm{~dB}$ ). Missing trials were discarded due to movement artifact. $\mathbf{C}$ Maximum DPOAE suppression during CAS in CBA/Cal mice, $\mathrm{a}^{+/+}$ mice, and $\mathrm{a}^{-/-}$mice, all in the unanesthetized condition. with mutant mice has shown that this slow enhancement effect is not mediated by the $\alpha 9 / \alpha 10$ ACh
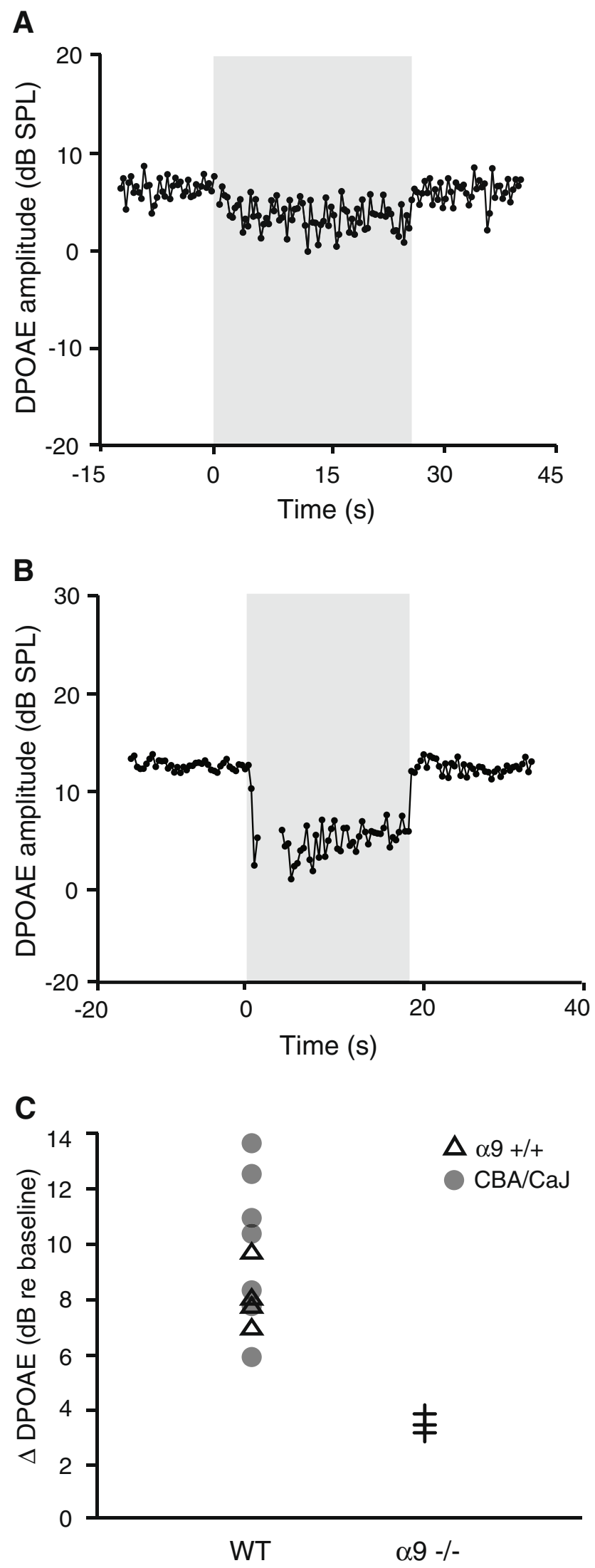
receptors on OHCs involved in MOC-mediated suppression (Maison et al. 2007). In $\alpha 9$ or $\alpha 10$ knockout mice, shocking the OC bundle produces only a slowonset enhancement of DPOAE amplitudes that slowly returns to baseline tens of seconds after shock-train offset. The origin of this slow enhancement effect, which is also visible in the neural output of the cochlea, is currently unknown (Maison et al. 2007), but the present results show that it is also present when MOC activity is evoked by CAS with or without anesthesia (Fig. 2C).

Shock-evoked MOC suppression peaks at $\sim 10 \mathrm{~dB}$ for primary frequencies in the middle of the cochlear spiral and declines monotonically toward the base and apex (Maison et al. 2007). This pattern mirrors the innervation density for MOC terminals on OHCs seen in most mammals, including mouse (Maison et al. 2003). Although the maximum CAS-evoked suppression was also close to $10 \mathrm{~dB}$ in the present study, the residual suppression in unanesthetized $\alpha 9$ knockout mice (3.6 $\mathrm{dB}$ on average) suggests that non-MOC mechanisms are contributing to the CAS effect and that the purely MOC-mediated suppression in mice is approximately $5.7 \mathrm{~dB}$. It is reasonable that CAS would only induce a fraction of the full inhibitory potential of this feedback system as it is only activating the contralaterally responsive MOC fibers, and this group constitutes less than one half of the MOC innervation to the ipsilateral ear (Brown 1993).

\section{Anesthesia and olivocochlear feedback in mammals}

The present results show that three common anesthetic regimens greatly attenuate the suppressive effect of CAS (Fig. 2). Given that most of the CAS-elicited suppression can be attributed to the MOC system (Fig. 4), the results also suggest that anesthesia attenuates the strength of the MOC reflex. However, CAS-evoked middle ear muscle activation can also suppress ipsilateral DPOAEs, and the relative thresholds for MOC and middle ear muscle activation depend on evoking stimulus properties, species, and anesthetic state. Several approaches have been used to isolate the contributions of MOC from those of the middle ear reflexes, including lowdose gentamicin (Smith et al. 1994), to selectively block the MOC synapses on OHCs (Guitton et al. 2004) or, as in the present study, targeted deletion of the OHC's ACh receptors.

The depressive effects of anesthesia reported here are consistent with previous reports in guinea pig where CASevoked DPOAE suppression of nearly $6 \mathrm{~dB}$ in the unanesthetized state was reduced to $\sim 1 \mathrm{~dB}$ after anesthetization with urethane and completely eliminated after anesthetization with pentobarbital (Guitton et al. 2004). Injection of low-dose gentamicin in this guinea pig study confirmed that the suppression in the unanesthetized animals was MOC-mediated. In contrast, in the unanesthetized rabbit, there is no detectable suppression of ipsilateral DPOAEs that cannot be ascribed to the middle ear muscle reflex (Whitehead et al. 1991). In awake humans, CAS-evoked suppression of DPOAEs is $\sim 1-2 \mathrm{~dB}$, with a white noise CAS of $60 \mathrm{~dB}$ SPL (Wagner et al. 2007). No studies of the effects of anesthesia on MOC reflex strength in humans have been published. MOC neurons have wide dynamic ranges: sound-evoked discharge rates increase monotonically with increasing stimulus level from threshold at $\sim 20 \mathrm{~dB}$ SPL up to $90 \mathrm{~dB}$ and beyond (Liberman 1988). Since the CAS in the guinea pig study was $50 \mathrm{~dB}$ SPL white noise, compared with $80 \mathrm{~dB}$ SPL white noise in the present study, the unanesthetized guinea pig study was unlikely to have characterized the maximum amount of DPOAE suppression induced by CAS.

Quantitative comparison between CAS-induced DPOAE suppression in different species, and even different studies in the same species, is complicated by the observation that small changes in primary frequency and/or level differences between the two primary tones can result in large differences in the amount of CAS-elicited suppression observed (Abdala et al. 2009; Wagner et al. 2007). This sensitivity to precise ipsilateral stimulus parameters probably arises from the complex interplay between two intracochlear sources of DPOAEs, with different phases, which, if differentially affected by MOC feedback, can amplify or attenuate the $\triangle$ DPOAE observed (Kalluri and Shera 2001). In practice, a CAS-evoked MOC assay based on DPOAEs tends to underestimate MOC reflex strength compared with one based on a compound neural potential evoked by a single ipsilateral tone (Puria et al. 1996); thus, the power of MOC feedback in turning down the gain of the cochlear amplifier is probably larger than suggested by the effects in Figures 2, 3, and 4.

\section{The olivocochlear reflex pathway} and its top-down modulation

While anesthesia clearly disrupts the central reflex pathway, our data cannot distinguish between its effects on (1) cochlear nucleus neurons that drive MOC neurons, (2) top-down midbrain and forebrain inputs that modulate MOC neurons, or (3) direct effects on MOC neurons themselves. Interactions between top-down modulatory systems and the MOC efferent system are particularly intriguing, yet poorly understood, perhaps because of the frequent use of anesthesia in animal studies. A small percentage of deep layer cortical projection neurons directly target MOC neurons in the ventral nucleus of the trapezoid body (Coomes and Schofield 2004; Feliciano and 
Potashner 1995), but the more prominent routes from the auditory cortex to the MOC neurons are mediated by polysynaptic projections via the inferior colliculus (Doucet et al. 2003; Thompson and Thompson 1993; Vetter et al. 1993) or cochlear nucleus (Schofield and Coomes 2005). Direct electrical stimulation of the auditory cortex in awake bats can modulate the cochlear microphonic in a frequencyspecific fashion, suggesting that the anatomical links between the corticofugal and olivocochlear control systems have a functional counterpart as well (Suga et al. 2002). Electrical stimulation of the inferior colliculus can also suppress DPOAE amplitudes, and the effects can be as large as those seen when directly shocking the MOC bundle at the floor of the fourth ventricle (Groff and Liberman 2003; Mulders and Robertson 2000; Scates et al. 1999).

Behaviorally, the engagement of corticofugal and olivocochlear descending systems has been associated with a number of adaptive perceptual benefits including enhanced recognition of speech in noise (de Boer and Thornton 2008; Dewson 1968; Kumar and Vanaja 2004; May et al. 2004; Messing et al. 2009; Micheyl and Collet 1996), increased auditory attention (de Boer and Thornton 2008; Harkrider and Bowers 2009), and recalibration of sound localization accuracy following manipulation of acoustic cues (Bajo et al. 2010; Irving et al. 2011). The neurophysiological mechanisms that govern the interplay between the activation of the two descending control systems and their associated effects on auditory perception are almost entirely unknown. Given the success of the mouse model in revealing the mechanisms behind efferent modulation of cochlear physiology and OHC micromechanics, there is reason to believe that genetically modified mice will also teach us a great deal about the modulation of MOC neurons by the corticofugal system. By adapting a traditional noninvasive measurement of the olivocochlear system to unanesthetized wild-type and knockout mice, we have taken some first steps in that direction.

\section{ACKNOWLEDGMENTS}

We are grateful to Dr. Douglas Vetter for generously sharing his $\alpha 9$-null and wild-type mice with us, to Drs. John Guinan, Jeremiah Cohen and Nao Uchida for helpful advice, and to Sara Anastas and John Thompson for animal care and technical assistance. Financial support was provided by NIDCD grants: P30 DC5029 (MCL), R01 DC0188 (MCL), R01 DC09836 (DBP), and R03 DC09488 (DBP).

\section{REFERENCES}

Abdala C, Mishra SK, Williams TL (2009) Considering distortion product otoacoustic emission fine structure in measurements of the medial olivocochlear reflex. J Acoust Soc Am 125:1584-1594
Avan P, Erre JP, Lima Da Costa D, Aran JM, Popelar J (1996) The efferent-mediated suppression of otoacoustic emissions in awake guinea pigs and its reversible blockage by gentimicin. Exp Brain Res 109:9-16

Bajo VM, Nodal FR, Moore DR, King AJ (2010) The descending corticocollicular pathway mediates learning-induced auditory plasticity. Nat Neurosci 13:253-260

Bauerle P, Von der Behrens W, Kossl M, Gaese BH (2011) Stimulusspecific adaptation in the gerbil primary auditory thalamus is the result of a fast frequency-specific habituation and is regulated by the corticofugal system. J Neurosci 31:9708-9722

Boyev KP, Liberman MC, Brown MC (2002) Effects of anesthesia on efferent-mediated adaptation of the DPOAE. J Assoc Res Otolaryngol 3:362-373

BRown MC (1993) Fiber pathways and branching patterns of biocytin-labeled olivocochlear neurons in the mouse brainstem. J Comp Neurol 337:600-613

Coomes DL, Schofield BR (2004) Projections from the auditory cortex to the superior olivary complex in guinea pigs. Eur J Neurosci 19:2188-2200

De Boer J, Thornton AR (2008) Neural correlates of perceptual learning in the auditory brainstem: efferent activity predicts and reflects improvement at a speech-in-noise discrimination task. J Neurosci 28:4929-4937

Dewson JH III (1968) Efferent olivocochlear bundle: some relationships to stimulus discrimination in noise. J Neurophysiol 31:122-130

Doucet JR, Molavi DL, Ryugo DK (2003) The source of corticocollicular and corticobulbar projections in area Te1 of the rat. Exp Brain Res 153:461-466

Feliciano M, Potashner SJ (1995) Evidence for a glutamatergic pathway from the guinea pig auditory cortex to the inferior colliculus. J Neurochem 65:1348-1357

Frisina RD, NEWman SR, ZHU XX (2007) Auditory efferent activation in CBA mice exceeds that of C57s for varying levels of noise. J Acoust Soc Am 121:El29-El34

Fuchs PA, MurRow BW (1992) A novel cholinergic receptor mediates inhibition of chick cochlear hair cells. Proc Biol Sci 248:35-40

Groff JA, Liberman MC (2003) Modulation of cochlear afferent response by the lateral olivocochlear system: activation via electrical stimulation of the inferior colliculus. J Neurophysiol 90:3178-3200

Guinan JJ JR (2006) Olivocochlear efferents: anatomy, physiology, function, and the measurement of efferent effects in humans. Ear Hear 27:589-607

Guitton MJ, Avan P, Puel JL, Bonfils P (2004) Medial olivocochlear efferent activity in awake guinea pigs. Neuroreport 15:1379-1382

HARkrider AW, Bowers CD (2009) Evidence for a cortically mediated release from inhibition in the human cochlea. J Am Acad Audiol 20:208-215

Harrison RV, Sharma A, Brown T, Jiwani S, James AL (2008) Amplitude modulation of DPOAEs by acoustic stimulation of the contralateral ear. Acta Otolaryngol 128:404-407

Henin S, Thompson S, Abdelrazeq S, Long GR (2011) Changes in amplitude and phase of distortion-product otoacoustic emission fine-structure and separated components during efferent activation. J Acoust Soc Am 129:2068-2079

Irving S, Moore DR, Liberman MC, Sumner CJ (2011) Olivocochlear efferent control in sound localization and experience-dependent learning. J Neurosci 31:2493-2501

Kalluri R, Shera CA (2001) Distortion-product source unmixing: a test of the two-mechanism model for DPOAE generation. J Acoust Soc Am 109:622-637

Kujawa SG, Liberman MC (2001) Effects of olivocochlear feedback on distortion product otoacoustic emissions in guinea pig. $\mathrm{J}$ Assoc Res Otolaryngol 2:268-278

Kumar UA, VAnaja CS (2004) Functioning of olivocochlear bundle and speech perception in noise. Ear Hear 25:142-146 
Lauer AM, MaY BJ (2011) The medial olivocochlear system attenuates the developmental impact of early noise exposure. J Assoc Res Otolaryngol 12:329-343

Liberman MC (1988) Physiology of cochlear efferent and afferent neurons: direct comparisons in the same animal. Hear Res 34:179-191

Liberman MC, Brown MC (1986) Physiology and anatomy of single olivocochlear neurons in the cat. Hear Res 24:17-36

Maison SF, Liberman MC (2000) Predicting vulnerability to acoustic injury with a noninvasive assay of olivocochlear reflex strength. J Neurosci 20:4701-4707

Maison SF, Luebke AE, Liberman MC, Zuo J (2002) Efferent protection from acoustic injury is mediated via alpha9 nicotinic acetylcholine receptors on outer hair cells. J Neurosci 22:10838-10846

Maison SF, Adams JC, Liberman MC (2003) Olivocochlear innervation in the mouse: immunocytochemical maps, crossed versus uncrossed contributions, and transmitter colocalization. J Comp Neurol 455:406-416

Maison SF, Vetter DE, Liberman MC (2007) A novel effect of cochlear efferents: in vivo response enhancement does not require alpha9 cholinergic receptors. J Neurophysiol 97:3269-3278

Maison SF, Usubuchi H, Vetter DE, Elgoyhen AB, Thomas SA, LIBERMAN MC (2011) In anesthetized mice, contralateral-noise effects on DPOAEs evoked by low-level primaries persist after elimination of olivocochlear and middle-ear reflexes. Association for Research in Otolaryngology. Baltimore, Maryland

May BJ, Budelis J, Niparko JK (2004) Behavioral studies of the olivocochlear efferent system: learning to listen in noise. Arch Otolaryngol Head Neck Surg 130:660-664

Messing DP, Delhorne L, Bruckert E, Braida LD, Ghitza O (2009) A nonlinear efferent-inspired model of the auditory system; matching human confusions in stationary noise. Speech Commun 51:668-683

Micheyl C, Collet L (1996) Involvement of the olivocochlear bundle in the detection of tones in noise. J Acoust Soc Am 99:1604-1610

Moulin A, Collet L, Duclaux R (1993) Contralateral auditory stimulation alters acoustic distortion products in humans. Hear Res 65:193-210

MukerJi S, Windsor AM, LeE DJ (2010) Auditory brainstem circuits that mediate the middle ear muscle reflex. Trends Amplif 14:170-191

Mulders WH, Robertson D (2000) Effects on cochlear responses of activation of descending pathways from the inferior colliculus. Hear Res 149:11-23

Puria S, Guinan IJ Jr, Liberman MC (1996) Olivocochlear reflex assays: effects of contralateral sound on compound action potentials versus ear-canal distortion products. J Acoust Soc Am 99:500-507

Rossel M, Loulier K, Feuillet C, Alonso S, Carroll P (2005) Reelin signaling is necessary for a specific step in the migration of hindbrain efferent neurons. Development 132:1175-1185

Scates KW, Woods CI, Azeredo WJ (1999) Inferior colliculus stimulation and changes in $2 f_{1}-f_{2}$ distortion product otoacoustic emissions in the rat. Hear Res 128:51-60
Schofield BR, CoOmes DL (2005) Projections from auditory cortex contact cells in the cochlear nucleus that project to the inferior colliculus. Hearing Res 206:3-11

Smith DW, Erre JP, Aran JM (1994) Rapid, reversible elimination of medial olivocochlear efferent function following single injections of gentamicin in the guinea pig. Brain Res 652:243-248

Sridhar TS, Liberman MC, Brown MC, Sewell WF (1995) A novel cholinergic slow effect of efferent stimulation on cochlear potentials in the guinea-pig. J Neurosci 15:3667-3678

Suga N, Xiao Z, Ma X, Ji W (2002) Plasticity and corticofugal modulation for hearing in adult animals. Neuron 36:9-18

Taranda J, Maison SF, Ballestero JA, Katz E, Savino J, Vetter DE, Boulter J, Liberman MC, Fuchs PA, Elgoyhen AB (2009) A point mutation in the hair cell nicotinic cholinergic receptor prolongs cochlear inhibition and enhances noise protection. PLoS Biol 7:e18

Thompson AM, Thompson GC (1993) Relationship of descending inferior colliculus projections to olivocochlear neurons. J Comp Neurol 335:402-412

Vetter DE, Saldana E, Mugnaini E (1993) Input from the inferior colliculus to medial olivocochlear neurons in the rat: a double label study with PHA-L and cholera toxin. Hear Res 70:173-186

Vetter DE, Liberman MC, Mann J, Barhanin J, Boulter J, Brown MC, Saffiote-Kolman J, Heinemann SF, Elgoyhen AB (1999) Role of alpha9 nicotinic ACh receptor subunits in the development and function of cochlear efferent innervation. Neuron 23:93-103

Vetter DE, Katz E, Maison S, Taranda J, Turcan S, Ballestero JA, Liberman MC, Elgoyhen AB, Boulter J (2007) The alpha10 nicotinic acetylcholine receptor subunit is required for normal synaptic function and integrity of the olivocochlear system. Proc Natl Acad Sci USA 104:20594-20599

Wagner W, Heppelmann G, Muller J, Janssen T, Zenner HP (2007) Olivocochlear reflex effect on human distortion product otoacoustic emissions is largest at frequencies with distinct fine structure dips. Hearing Res 223:83-92

Whitehead ML, Martin GK, Lonsburz-Martin BL (1991) Effects of the crossed acoustic reflex on distortion-product otoacoustic emissions in awake rabbits. Hear Res 51:55-72

WiEDERHOLD ML, KIANG NY (1970) Effects of electric stimulation of the crossed olivocochlear bundle on single auditory-nerve fibers in the cat. J Acoust Soc Am 48:950-965

Wienisch M, Blauvelt D, Sato TF, Murthy VN (eds) (2011) Twophoton imaging of neural activity in awake, headfixed mice. Humana, New York

Winer JA (2006) Decoding the auditory corticofugal systems. Hear Res 212:1-8

YAN J, Zhang Y, Ehret G (2005) Corticofugal shaping of frequency tuning curves in the central nucleus of the inferior colliculus of mice. J Neurophysiol 93:71-83

Zhu X, Vasilyeva ON, Kim S, Jacobson M, Romney J, Waterman MS, Tuttle D, Frisina RD (2007) Auditory efferent feedback system deficits precede age-related hearing loss: contralateral suppression of otoacoustic emissions in mice. J Comp Neurol 503:593-604 